\title{
THRESHOLD DYNAMICS FOR SHAPE RECONSTRUCTION AND DISOCCLUSION
}

\author{
Selim Esedoḡlu*
}

\author{
Steven Ruuth ${ }^{\dagger}$
}

Richard Tsai ${ }^{\ddagger}$

April 3, 2005

\begin{abstract}
We propose a very efficient numerical algorithm for minimizing certain curvature dependent functionals that appear in a variety of well known variational models of image processing and computer vision. It has many applications, such as image inpainting, image segmentation, and surface fairing in computer graphics. The proposed algorithm is generalized to a level set algorithm that has better resolution while keeping the same formal complexity. As an example, we apply our technique to a shape reconstruction problem based on Euler's elastica.
\end{abstract}

\section{Introduction}

Many important models of image processing and computer vision involve curvature dependent functionals. In segmentation, the Kass, Witkin, Terzopoulos method [17] of snakes originally calls for minimizing an active contour energy that involves integrating curvature squared along the curve. In segmentation with depth, the 2.1D Sketch model of Nitzberg, Mumford, and Shiota [24] involves the integral of a function of the curvature along the free discontinuity set. In the image inpainting application of Bertalmio et. al. [4], Chan et. al. [7] proposed generating the missing image information in the inpainting domain by minimizing Euler's elastica energy along image isophotes; see also [1, 3, 5] on image inpainting and $[6,12]$ for other curvature dependent functional approaches to this problem. Finally, in computer graphics, curvature dependent functionals have been proposed for surface denoising and smoothing $[9,28,29]$.

*Department of Mathematics, UCLA. Los Angeles, CA 90095. Email: esedoglu@math.ucla.edu. Research supported in part by NSF grant DMS-0410085, NSF grant DMS-9973341, NIH grant P20 MH65166, ONR grant N00014-03-1-0888, and ONR grant N00014-02-1-0720.

${ }^{\dagger}$ Department of Mathematics, Simon Fraser University. Burnaby, British Columbia, V5A 1S6, Canada. Email: sruuth@sfu.ca. Research supported in part by a grant from NSERC Canada.

$\ddagger$ Department of Mathematics, University of Texas at Austin, TX 78712. Email: ytsai@math.utexas.edu. 


\section{Background}

One of the most successful techniques for minimizing variational models in image processing that involve unknown contours has been the level set method of Osher and Sethian [26]. Once the energies in question are written in terms of a level set representation for the unknown curves, optimality conditions (Euler-Lagrange equations) can be obtained in terms of the level set function, and a gradient descent procedure can be carried out. This involves solving non-linear, degenerate, fourth order parabolic PDE and can be computationally very expensive. Another popular approach is generally referred to as the phase field method, in which the unknown curve will be computed and identified as the smooth transition region between two order parameter. Typically in this formulation, a stiff potential appears as part of the energy functional. The stiffness of the system and the need to resolve the narrow transition layer result in a stringent stability condition for the corresponding numerical algorithms.

In this paper we propose an efficient algorithm based on threshold dynamics (also known as diffusion generated motion) that seems to bypass the computational bottlenecks of the aforementioned approaches. The idea is to alternate the solution of a linear parabolic PDE, such as the heat equation, and thresholding to generate geometric motion of interfaces. The original idea is due to Merriman, Bence, and Osher [20, 21, 22], who proposed a technique for approximating the motion by mean curvature of an interface by alternating the solution of the heat equation (i.e. convolution by the Gaussian kernel) and thresholding. Convergence was proved by Evans [14], and by Barles and Georgelin [2]. There have been various generalizations of their method [19, 30, 31, 32], including to other curvature dependent velocities, and a highly accurate version was developed by Ruuth in [27]. These offer an alternative to level set based techniques that require the solution of nonlinear second order equations.

Motivated by the Merriman, Bence, Osher scheme, and also partially by [15, 33], recently Esedoglu and Tsai proposed a technique for minimizing the piecewise constant versions of the Mumford-Shah segmentation functional that were introduced by Chan and Vese $[8,35]$. This new algorithm involves alternating the solution of a linear parabolic PDE and simple thresholding. It leads to a very efficient minimization of Chan and Vese's Mumford-Shah energies.

In this paper, we propose a efficient thresholding algorithm for minimizing the Euler's elastica energy [7]. The energy is of the form:

$$
\int_{C} \kappa^{2}+\beta d \sigma .
$$

where $C$ is a curve, $d \sigma$ is the length element, and $\kappa$ is the curvature of the curve. This type of term typically appears in more elaborate models such as the 2.1D sketch model of [24].

Central to our approach is the recent results of Grzibovskis and Heintz [16], who have discovered a convolution-thresholding scheme for Willmore flow. Willmore flow is gradient descent for the following energy:

$$
W(\Sigma)=\int_{\partial \Sigma} H^{2} d \sigma
$$


where $\partial \Sigma$ is the boundary of $\Sigma \subset \mathbf{R}^{N}$ (i.e. a closed hypersurface) and $H$ is its mean curvature. When $\Sigma$ is a region in the plane, energy (2) reduces to (1) with $\beta=0$ and $C=\partial \Sigma$.

\section{The proposed algorithms}

As a first step in bringing threshold dynamics to bear upon higher order image processing and vision models, we use energy (1) to reconstruct missing (or occluded) parts of boundaries of shapes. Let $D$ be the image region, and let the given image be binary, i.e. it is a shape: $f(x)=\mathbf{1}_{\Omega}(x)$, where $\Omega \subset D$. Let $\tilde{D}$ be a subdomain of $D$ with nice boundary where image (i.e. shape) information is known to be missing. To generate the (possibly) missing part of the observed shape $\Omega$ in $\tilde{D}$, one can use the following model, which is a much simplified version of the Nitzberg-Mumford-Shiota (NMS) model:

$$
\min _{\Sigma \text { s.t. } \Sigma \cap \tilde{D}^{c}=\Omega \cap \tilde{D}^{c}} \int_{\partial \Sigma} \kappa_{\Sigma}^{2}+\beta d \sigma .
$$

A level set based implementation of the NMS model was carried out in [36], and a diffuse interface (i.e. phase field) implementation appears in [11] following previous work by March and Dozio in [18].

To minimize (3) we alternate the dynamics of Grzibovskis and Heintz that decreases $\int_{\partial \Sigma} \kappa^{2} d \sigma$, and the standard MBO scheme that decreases $\int_{\partial \Sigma} \beta d \sigma$. This involves, in particular, deriving the two dimensional analogue (i.e the analogue for planar curves) of the algorithm in [16], which is originally formulated for two dimensional surfaces in $\mathbb{R}^{3}$. Also, we keep part of the boundary $\partial \Sigma$ that lies in $\tilde{D}^{c}$ fixed.

The algorithm has the following form: Let $G_{t}(x)$ be the Gaussian kernel in two dimensions:

$$
G_{t}(x)=\frac{1}{4 \pi t} e^{-\frac{|x|^{2}}{4 t}}
$$

. Fix a number $\alpha \in(0,1)$, and a $\delta t>0$. Starting with an initial guess $\Sigma_{0} \subset D$ with $\Sigma \cap \tilde{D}^{c}=\Omega \cap \tilde{D}^{c}$, repeat the following steps for $n=1,2,3, \ldots$ :

1. A step of Grzibovskis and Heintz's algorithm: Set

$$
\begin{aligned}
\Gamma_{1}=\left\{x: 2 \alpha G_{\sqrt{\delta t}} * \mathbf{1}_{\Sigma_{n}}(x)\right. \\
\left.-2 G_{\alpha^{2} \sqrt{\delta t}} * \mathbf{1}_{\Sigma_{n}}(x)+1-\alpha \geq 0\right\} .
\end{aligned}
$$

2. A step of standard MBO algorithm: Set

$$
\Gamma_{2}=\left\{x: G_{\beta \delta t} * \mathbf{1}_{\Gamma_{1}}(x) \geq \frac{1}{2}\right\} .
$$

3. Fidelity step: Set

$$
\Sigma_{n+1}=\left(\Gamma_{2} \cap \tilde{D}\right) \cup(\Omega \backslash \tilde{D}) .
$$


where $\mathbf{1}_{S}(x)$ denotes the characteristic (i.e. indicator) function of a set $S$. Each step of the algorithm given above can be generalized so that the convolutions are performed with any rotationally symmetric kernel. In our computations, we use a scaled version of the compactly supported kernel

$$
K(r)=C_{0} \cdot 1_{[-1,1]} e^{\frac{1}{1-r^{2}}}
$$

in Step 1 as an attempt to eliminate the effect of the exponential tail of $G_{t}(x)$ when two interfaces are close to each other. The convolutions are implemented via the fast Fourier transform.

It is actually possible to generalize the above algorithm so that the interface is represented by a continuous function, while maintaining its simplicity and efficiency. Representing the interface by a continuous (or better) function has the important advantage that the geometry of the interface is not degraded after the thresholding step is performed: the characteristic function of a set (e.g. one that only takes one of the two values 0 or 1 at every grid point) does not allow for interpolation and thus cannot attain subgrid resolution. The resulting algorithm is more similar to but still very distinct from a conventional level set implementation of the geometric motion involved. We observe that if we use a Lipschitz continuous level set function $\phi(x)$ such that $\Omega=\{\phi \geq 0\}$, then the characteristic function $1_{\Omega}(x)$ can be represented as $H(\phi)$, where $H(\xi): \mathbb{R} \rightarrow \mathbb{R}$ is the conventional Heaviside function:

$$
H(\xi)= \begin{cases}0 & \text { if } x \leq 0 \\ 1 & \text { if } x>0\end{cases}
$$

Hence, in the convolution steps, the convolution of a kernel $G_{t}$ with $1_{\Omega}$ is equivalent to $G_{t} * H(\phi)$. Let $u$ be the resulting function. We observe that $u$ is continuous and the thresholding step is to project the values of $u$ onto the 0,1 ; that is $u(x)=H(u(x)-0.5)$. Therefore, instead of the projection, we can perform a fast reinitialization step so that $u(x)-1 / 2$ is reshaped into the signed distance function to $\partial \Omega$. This enable us to perform the convolution step as stated again. In summary, a continuous interface version of the diffusion generated motion can be written as:

1. Convolution: $u(x)=K_{\alpha} * H\left(\phi^{(n)}(x)\right)$

2. Reinitialize: $\phi^{(n+1)}(x)=\operatorname{reinit}(u-/ 12)$.

The real difference between the two versions of our algorithm lies in their discretizations on uniform Cartesian grids. The order of accuracy of a typical quadrature on a Cartesian grid for the integration of a piecewise smooth function is first order in the mesh size $h$. In [10], Engquist et al. investigated a regularization technique related to the Heaviside function $H$. According to their result, it is possible to obtain second order accuracy $\left(\mathcal{O}\left(h^{2}\right)\right)$ in the quadrature for the convolution step, if one regularizes $H(x)$ by $H_{\epsilon(h, \phi(x))}(x)$ with a suitably chosen $\epsilon(h, \phi(x))$ defined in the paper. $H_{\epsilon}(x)=H(x)$ for $|x|>\epsilon / 2$ and is $1 / 2+x / \epsilon$ for $|x| \leq \epsilon / 2$. The reinitialization step keeps a locally $\mathcal{O}\left(h^{2}\right)$ approximation to $\partial \Omega^{(n+1)}$, and formally keeps the complexity of the original method if one uses a fast reinitialization technique such as one of the fast sweeping schemes, see [34] and the references therein. We point out, however, that this approach does not improve the formal analytical error that comes from the asymptotics: it does not improve on the convergence rate with respect to the time variable of standard thresholding based 
algorithms such as the Merriman, Bence, Osher scheme. On the other hand, accuracy in time is less of an issue for the applications considered in this paper, since the accuracy of the dynamics is not important as long as an accurate stationary state is attained.

\section{Numerical Results}

Numerical results with our proposed algorithms are very encouraging. Here we present our results on the two examples that have been extensively used in the literature. In our simulations, we used a compactly supported kernel and the continuous interface version of our algorithm. In our simulations, the algorithm (and its level set version) requires about 15 or 20 iterations for computations that typically take tens of thousands of iterations with standard methods.

Figure 1 shows an experiment where the missing part of a disk is to be reconstructed. The occluding region (i.e. region $\tilde{D}$ in model 3 ) is the upper left quarter square. The left hand image shows the recovered boundary when only the length of the boundary contour is minimized. The right hand image shows the result obtained when Euler's elastica model (3) is used. In both cases, the computation was started with random initial data in domain $\tilde{D}$, and took only about 20 iterations to complete.

Figure 2 shows the same experiment as in Figure 1, this time using a "broken bar" as the given image, and the middle square shown with random initial data as the occluding region $\tilde{D}$. Once again, the left image shows the completion obtained by minimizing only the length of the contour, whereas the right hand image shows the result of minimizing Euler's elastica model (3). As is well known, the elastica model prefers to connect junctions on either side of the occluding region, despite the wide separation. The algorithm proposed in this paper requires only about 15 iterations to reach the fixed points shown, starting from random initial data in $\tilde{D}$.

There is an important point that needs to be discussed concerning the numerical example of Figure 2. The initial curve in this computation has two connected components. During the course of the evolution these two parts of the curve get close to each other and at some point are observed to merge into a single connected componenet. This merger involves a topological change. Exactly at the time of this topological change, there are singularities in the curve that from a naive point of view would suggest that the configuration has infinite energy. (The correct interpretation would be that the energy of this configuration is the limit of energies of smooth approximating configurations, which would be finite). Immediately after the topological change, as the curve - which now consists of one connected component - starts to straighten itself out, corresponding energies can be arbitrarily high. This observation raises the possibility that in fact the topological change might not take place if the computation is repeated using more localized (i.e. narrower) kernels. In other words, the merger that we observed might be due to the fact that the computation was carried out with a relatively wide kernel, giving us rather diffuse interfaces. Clearly, this is a very important issue that deserves to be investigated further. 

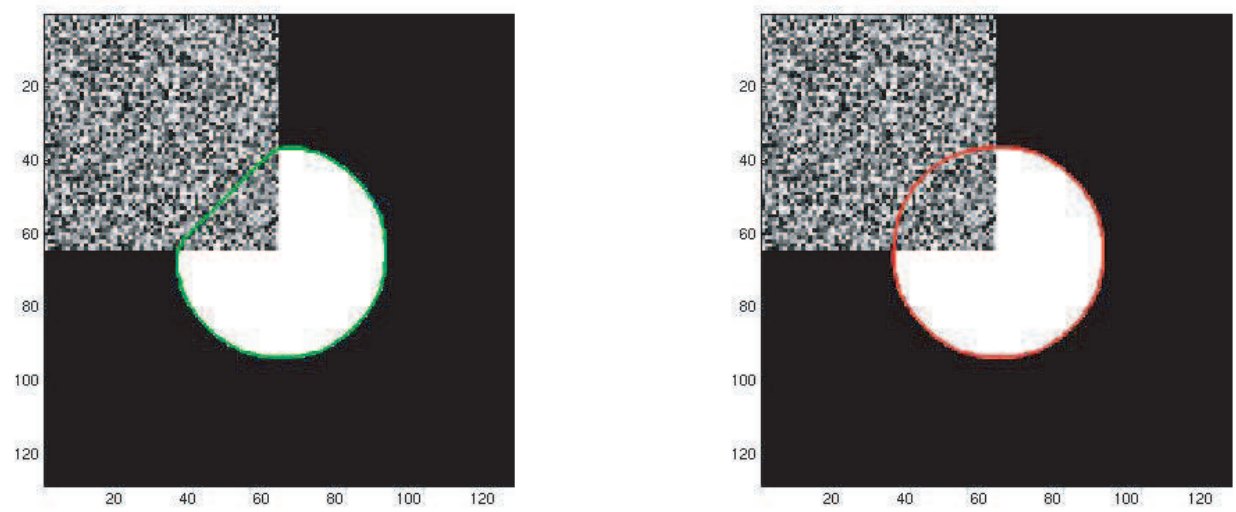

Figure 1: Reconstructed edge contour by minimizing length (left image) and Euler's elastica based model (3). The upper left hand square is the occluding (or damaged) region $\tilde{D}$ in the model. About 20 iterations of our proposed algorithm were required.

\section{Conclusion}

We introduced an efficient algorithm for minimizing shape functionals related to the Euler's Elastica model. Our algorithm uses convolutions of the characteristic function of a shape with a smooth kernel to generate a consistent approximation to the front velocity. We further proposed a continuous interface algorithm for diffusion generated motion that has the same formal complexity in each iteration as our first algorithm. This level set method preserves more geometrical information of the shape and thus improves the resolution of the first algorithm. Finally, we demonstrated that our proposed algorithm yields results resembling those computed by the exisiting level set methods. A detailed analysis on the scaling and convergence of our algorithm and generalization will be reported by the authors in a forthcoming paper.

\section{References}

[1] Ballester, C.; Bertalmio, M.; Caselles, V.; Sapiro, G.; Verdera, 1 J. Filling-in by joint interpolation of vector fields and gray levels. IEEE Transactions on Image Processing. 10:8 (2001), pp. 1200-1211.

[2] Barles, G.; Georgelin, C. A simple proof of convergence for an approximation scheme for computing motions by mean curvature. SIAM J. Numer. Anal. 32:2 (1995), pp. $484-500$.

[3] Bertalmio, M.; Bertozzi, A.; Sapiro, G. Navier-Stokes, fluid dynamics, and image and video inpainting. IEEE Computer Vision and Pattern Recognition (CVPR), Hawaii. December 2001.

[4] Bertalmio, M.; Sapiro, G.; Caselles, V.; Ballester, C. Image inpainting. SIGGRAPH 2000, Computer Graphics Proceedings, pp. 417 - 424. 

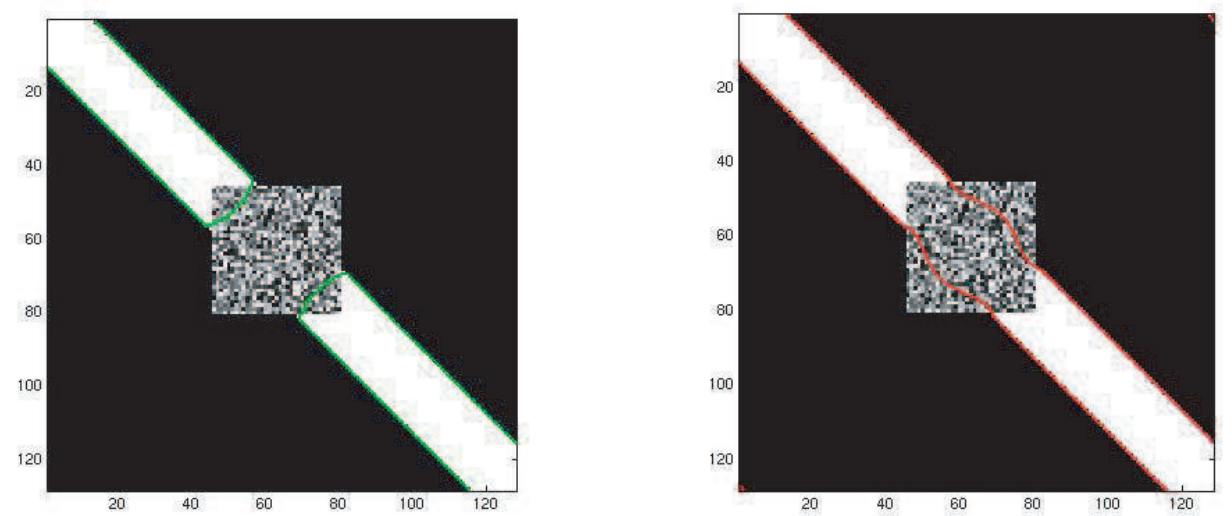

Figure 2: Reconstructed edge contour by minimizing length (left image) and Euler's elastica based model (3). The middle square represents the damaged region $\tilde{D}$ in the model. Only about 15 iterations of our algorithm were required.

[5] Chan, T. F.; Shen, J. Mathematical models for local non-texture inpaintings. SIAM Journal on Applied Mathematics. 61:4, pp. 1019-1043.

[6] Chan, T. F.; Shen, J. Non-texture inpainting by curvature-driven diffusions. Journal of Visual Communication and Image Representation. 12:4 (2001), pp. 436-449.

[7] Chan, T. F.; Shen, J.; Kang, S. Euler's elastica and curvature-based inpainting. SIAM J. Appl. Math. 63:2 (2002), pp. 564 - 592.

[8] Chan, T. F.; Vese, L. A. Active contours without edges. IEEE Transactions on Image Processing. 10:2 (2001), pp. 266 - 277.

[9] Clarenz ,U.; Diewald, U.; Rumpf, M. Anisotropic diffusion in surface processing. Proceedings of Visualization 2000. Editors: T. Ertl, B. Hamann, and A. Varshney. (2000), pp. $397-405$.

[10] Engquist, B.; Tornberg, A-K.; Tsai, Y-H. Dirac- $\delta$ functions in level set methods. CAM Report 04-16, UCLA, 2003. Under review.

[11] Esedoglu, S.; March, R. Segmentation with depth but without detecting junctions. Journal of Mathematical Imaging and Vision. 18 (2003), pp. 7-15.

[12] Esedoglu, S.; Shen, J. Digital inpainting using the Mumford-Shah-Euler image model. European Journal of Applied Mathematics. 13 (2002), pp. 353-370.

[13] Esedoglu, S.; Tsai, R. Threshold dynamics for the piecewise constant MumfordShah functional. UCLA CAM Report 04-63 (October 2004).

[14] Evans, L. C. Convergence of an algorithm for mean curvature motion. Indiana University Mathematics Journal. 42 (1993), pp. 553 - 557.

[15] Gibou, F.; Fedkiw, R. A fast hybrid k-means level set algorithm for segmentation. Stanford Technical Report (November 2002). Accepted in Proceedings of the 4th Hawaii International Conference on Statistics, Mathematics, and Related Fields. Honolulu, January 2005. 
[16] Grzibovskis, R.; Heintz, A. A convolution-thresholding scheme for the Willmore flow. Preprint, October 13, 2003.

[17] Kass, M.; Witkin, A.; Terzopoulos, D. Snakes: Active contour models. International Journal of Computer Vision. 1 (1988), pp. 321 - 331.

[18] March, R.; Dozio, M. A variational method for the recovery of smooth boundaries. Image and Vision Computing. 15 (1997), pp. 705-712.

[19] Mascarenhas, P. Diffusion generated motion by mean curvature. UCLA CAM Report 92-33 (July 1992).

[20] Merriman, B.; Bence, J.; Osher, S. J. Diffusion generated motion by mean curvature. Proceedings of the Computational Crystal Growers Workshop, pp. 73-83. Editor: Jean Taylor. AMS, Providence, Rhode Island, 1992.

[21] Merriman, B.; Bence, J. K.; Osher, S. J. Diffusion generated motion by mean curvature. UCLA CAM Report 92-18 (April 1992).

[22] Merriman, B.; Bence, J. K.; Osher, S. J. Motion of multiple junctions: a level set approach. J. Comput. Phys. 112:2 (1994), pp. $334-363$.

[23] Mumford, D.; Shah, J. Optimal approximations by piecewise smooth functions and associated variational problems. Comm. Pure Appl. Math. 42 (1989), pp. 577 685 .

[24] Nitzberg, M.; Mumford, D.; Shiota, T. Filtering, segmentation, and depth. Lecture Notes in Computer Science 662. Springer-Verlag (1993).

[25] Osher, S.; Fedkiw, R. Level set methods and dynamic implicit surfaces. Applied Mathematical Sciences, 153. Springer-Verlag, New York, 2003.

[26] Osher, S.; Sethian, J. Fronts propagating with curvature-dependent speed: algorithms based on Hamilton-Jacobi formulations. Journal of Computational Physics. 79:1 (1988), pp. 12 - 49.

[27] Ruuth, S. J. Efficient algorithms for diffusion-generated motion by mean curvature. J. Comput. Phys. 144:2 (1998), pp. 603 - 625.

[28] Tasdizen, T.; Whitaker, R.; Burchard, P.; Osher, S. Geometric surface smoothing via anisotropic diffusion of normals. Proceedins of IEEE Visualization 2002, pp. $125-132$.

[29] Tasdizen, T.; Whitaker, R.; Burchard, P.; Osher, S. Geometric surface processing via normal maps. ACM Transactions on Graphics (2003).

[30] Ruuth, S. J. A diffusion generated approach to multiphase motion. J. Comput. Phys. 145:1 (1998), pp. 166 - 192.

[31] Ruuth, S. J.; Merriman, B. Convolution generated motion and generalized Huygens' principles for interface motion. SIAM J. Appl. Math. 60:3 (2000), pp. 868 890.

[32] Ruuth, S. J.; Merriman, B. Convolution-thresholding methods for interface motion. J. Comput. Phys. 169:2 (2001), pp. 678 - 707. 
[33] Song, B.; Chan. T. A fast algorithm for level set based optimizations. UCLA CAM Report 02-68 (December 2002).

[34] Tsai, Y.-H. R.; Cheng, L.-T.; Osher, S.; Zhao H.-K. Fast sweeping methods for a class of Hamilton-Jacobi equations SIAM J. Numer. Anal. 41:2 (2003), pp. 673694.

[35] Vese, L.; Chan, T. F. A multiphase level set framework for image segmentation using the Mumford and Shah model. IJCV 50:3 (2002), pp. 271 - 293.

[36] Zhu, W.; Chan, T. F.; Esedoglu, S. Segmentation with depth: A level set approach. UCLA CAM Report 04-49 (August 2004). 